\title{
Le rôle des mouvements associatifs et des ONG en faveur du rapprochement communautaire à Chypre
}

The role of associations and NGOs in promoting community relations in Cyprus

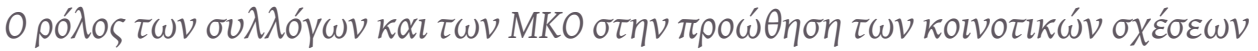
$\sigma \tau \eta v$ Kú $\rho_{\rho}$

\section{Alexandre Lapierre}

\section{(2) OpenEdition}

\section{Journals}

Édition électronique

URL : https://journals.openedition.org/ceb/14118

DOI : $10.4000 /$ ceb. 14118

ISSN : 2261-4184

Éditeur

INALCO

Édition imprimée

ISBN : 9782858313341

ISSN : 0290-7402

\section{Référence électronique}

Alexandre Lapierre, «Le rôle des mouvements associatifs et des ONG en faveur du rapprochement communautaire à Chypre », Cahiers balkaniques [En ligne], 46 | 2020, mis en ligne le 25 février 2020, consulté le 06 juillet 2021. URL : http://journals.openedition.org/ceb/14118 ; DOI : https://doi.org/ $10.4000 /$ ceb. 14118

\section{cc) (1) 8}

Cahiers balkaniques est mis à disposition selon les termes de la Licence Creative Commons Attribution - Pas d'Utilisation Commerciale 4.0 International. 


\section{Le rôle des mouvements associatifs et des ONG en faveur du rapprochement communautaire à Chypre \\ The role of associations and NGOs in promoting community relations in Cyprus

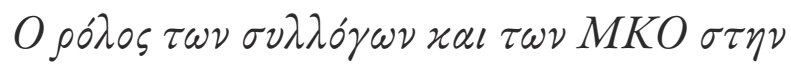

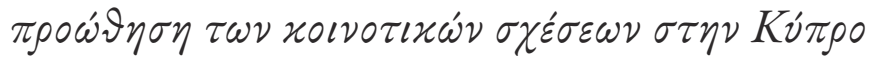

Alexandre Lapierre

CREE-Inalco

L'île de Chypre vit divisée depuis 1974 sans que la succession de négociations au sommet, qui se poursuivent actuellement, ne soit parvenue à apporter une solution au problème. La partition de l'île a provoqué au sein de chacune des deux communautés majoritaires, grecque et turque, une modification progressive des logiques identitaires, mettant en œuvre des dynamiques de rapprochement, certes fragiles, mais qui s'expriment dans certains discours politiques et surtout au sein de la société civile. Le phénomène est particulièrement visible depuis 2003, année qui marque un tournant majeur dans le dialogue intercommunautaire. Elle signe en effet le rétablissement d'une circulation de part et d'autre du $35^{\mathrm{e}}$ parallèle, et donc la perspective de nouveaux échanges entre les deux communautés.

Il est intéressant dans ce contexte de se pencher sur l'affirmation progressive de la société civile parallèlement à l'évolution des discours officiels dans le cadre $\mathrm{du}$ rapprochement communautaire. Nous pouvons en effet constater que les mouvements associatifs favorables à ce rapprochement se sont d'abord exprimés en réaction aux choix politiques qui, après 1974 , tendaient à cristalliser la position de chaque communauté, avant de susciter des dynamiques propres à encourager un processus politique. 
CAHIERS BALKANIQUES

96 Politique et sociétés à Chypre aujourd'hui

\section{Une construction par réaction}

Avant d'aller plus loin dans notre réflexion sur le rapprochement communautaire et le rôle qu'y tiennent les mouvements associatifs, il convient de se pencher sur le « chypriotisme » qui émerge après les événements de 1974 et la partition de l'île.

\section{L'émergence du chypriotisme au sein de la communauté grecque}

Du côté chypriote grec, l'Enosis n'est plus à l'ordre du jour, le mythe du rattachement à la Mère-Patrie s'est émoussé dans la plupart des esprits et les Chypriotes grecs sont de plus en plus critiques à l'égard de la Grèce qui les a abandonnés à l'intervention turque qu'elle a provoquée. Le chypriotisme s'était manifesté chez les Chypriotes grecs de diverses manières avant 1974, souvent dans le contexte du parti communiste, mais il était étouffé par la force idéologique et pédagogique du nationalisme hellénique et souvent contraint à insister sur la couleur locale des us et coutumes chypriotes pour marquer son originalité ${ }^{\text {. }}$ Les événements de 1974 ont renforcé les partisans de ce mouvement en faisant prendre conscience à certains Chypriotes, grecs ou turcs, de leur «différence » par rapport aux Grecs et aux Turcs. La création de l'Association pour la Nouvelle Chypre en mars 1975 dans la mouvance de l'AKEL, héritière de la tradition de la gauche chypriote à chercher une alliance des communautés, traduit un désir politique de se démarquer de la culture et de l'identité enosiste grecque ${ }^{2}$. L'AKEL en effet, dès 1975, appelle au rapprochement des frères « chypriotes » :

Chypriotes, Grecs ou Turcs, venons ensemble et effaçons toutes les passions et la haine. Marchons ensemble pour retirer toutes les troupes étrangères de notre île. Formons une fédération bizonale, avec un gouvernement central fort, et apportons la paix à notre île... Rétablissons la démocratie, la prospérité et le bonheur pour tous les frères chypriotes ${ }^{3}$.

L'Association pour la Nouvelle Chypre naît de réunions informelles entre neuf Chypriotes grecs issus des milieux universitaires et économiques qui cherchent à renforcer l'indépendance de Chypre. Après juillet 1974, l'objectif de ses

1. Calotychos, 1998, p. 1-31.

2. Attitude parfois poussée à l'extrême, l'AKEL, leader du mouvement chypriotiste, étant allé jusque à rejeter par principe tout ce qui pouvait se rattacher à «l'impérialisme grec », ibid. p. 93.

3. AKEL, Kibrıslitürkler ve Kıbrıslırumların Ortak Mücadeleleri, Nicosia, 1975, p. 141, cité dans Hamit, 2009, p. 20. 
membres fondateurs est de réagir face à la grave situation de l'île. Leurs premières motivations sont donc de réfléchir aux possibles solutions de la crise, et de proposer des réponses aux réfugiés. Leur volonté est de projeter Chypre dans son avenir et d'éviter le repli sur un passé douloureux. Très rapidement cependant l'Association pour la Nouvelle Chypre effectue un travail d'introspection sur sa communauté. Consciente des erreurs des politiques menées avant 1974, elle en mesure les dangers: la division communautaire a été exacerbée au gré de l'intransigeance nationaliste, l'île est menacée d'une division définitive, que la Turquie encourage, en tentant de dissoudre la République de Chypre. Ces quelques textes de la fin de l'été 1975 sont particulièrement éclairants. «Prenons conscience de nos erreurs », dit le premier extrait :

Maintenant que les larmes sont sèches, maintenant que la colère et le désespoir sont passés, nous devons réfléchir : nous avions une existence sereine et sans souci et nous nous taisions. Maintenant, nous payons pour ce silence. Nous, la majorité silencieuse, nous devons fouiller dans nos esprits et nos consciences pour réaliser le réveil subit de ces sept jours.

Un second extrait invite les Chypriotes à ne compter que sur eux-mêmes :

[... L] de danger de la partition de Chypre ou la dissolution de notre État est imminente et la responsabilité pour l'éviter incombe à nous, les Chypriotes, et aucun pays en dehors de Chypre ne peut aider effectivement, soit parce qu'il n'en a pas la puissance [= la Grèce], soit parce que ses intérêts ne s'identifient pas toujours avec les nôtres $[=\text { la Turquie }]^{4}$.

L'Association pour la Nouvelle Chypre ne veut pas supprimer les différences ethniques, religieuses ou culturelles, mais simplement faire passer la citoyenneté commune au premier plan. Sa structure est au départ très modeste, son discours se rapproche des idées progressistes de l'AKEL et ses premiers membres appartiennent aux élites intellectuelles et professionnelles de gauche et du centre gauche. Ces personnes ayant suivi des études supérieures en Grande-Bretagne ou aux États-Unis affirment souvent que de ne pas avoir étudié en Grèce leur a fait voir différemment la cohabitation multiethnique. Elles ne veulent pas créer un parti politique, mais plutôt agir comme un think tank ou un groupe de pression.

Dans un contexte où les partis de droite sont décrédibilisés, l'Association pour la Nouvelle Chypre jouit dès ses premières années d'un grand soutien de

4. Mavratsas, 2007, p. 717-737. 
98 Politique et sociétés à Chypre aujourd'hui

l'opinion publique. La population déçue par la Grèce est d'autant plus sensible à son discours insistant sur l'identité chypriote. L'Association rencontre ainsi un succès certain dans les trois défis que sont la promotion du rapprochement communautaire, la préservation de la République de Chypre, et le renforcement de la démocratie, atteinte par le coup d'État. Ses rapports avec la communauté turque sont cependant inexistants dans les premières années, mais elle parvient à sensibiliser la communauté grecque aux souffrances de l'autre communauté et à établir une distinction entre les Chypriotes turcs et les Turcs que l'opinion commune assimilait et considérait logiquement comme une même menace.

Cette action visant à distinguer l'identité politique et la culture propre du pays est assez difficilement comprise, tant l'idée de rattachement à la Grèce est présente dans les manifestations publiques. À l'époque, les principales fêtes officielles sont calquées sur le calendrier des commémorations grecques. Le 25 mars en mémoire de la révolution grecque contre les Ottomans, et le 28 octobre en mémoire du Grand Non [To $\left.\mu \varepsilon \gamma \alpha \dot{\lambda} 0^{\prime} O \chi \iota\right]$, opposé à l'ultimatum italien suivi de la victoire de la Grèce contre l'Italie en 1941, sont ainsi célébrés avec une importance variable selon les appartenances politiques, mais c'est seulement à partir de 1979 que l'indépendance de Chypre est officiellement fêtée. Il en va de même pour le drapeau chypriote qui longtemps n'a pas d'existence sur les bâtiments officiels, supplanté par le drapeau grec alors qu'il existe depuis l'indépendance et que ses codes de couleurs ont été choisis pour ne choquer aucune sensibilité.

Les membres de l'Association pour la Nouvelle Chypre font en outre l'objet de nombreuses pressions dans leur milieu professionnel ainsi que dans les médias, en raison de leur remise en question non voilée de l'enosis. Toutefois, grâce à sa médiatisation, l'Association parvient à imposer les symboles de la nation chypriote et à poser les bases d'un retour au dialogue communautaire, elle constitue de ce fait un des grands acteurs du mouvement chypriotiste en cours de formation. Elle ne parvient cependant pas à lever les confusions entre identité cultuelle et identité nationale issues de l'Empire ottoman et perpétuées depuis lors. Son action marque certes les esprits, mais n'a pas forcément de répercussions sur la politique gouvernementale, notamment en matière d'éducation. Après la victoire du PASOK en Grèce en 1981, l'Association perd de sa représentativité médiatique, car l'opinion publique est plus encline à se rapprocher de la Grèce. Elle n'en continue pas moins à organiser des manifestations autour du chypriotisme et à inviter à ses instances des représentants de la communauté turque. Elle reste un réservoir d'idées pour l'avenir sans devenir un mouvement politique.

À Chypre Nord, dès les premières années, Ankara pratique une politique de colonisation mal vécue par les Chypriotes turcs dans un contexte économique particulièrement rationné marqué par l'isolement international. La Turquie 
assortit ce mouvement d'une politique d'affirmation identitaire dont les manifestations les plus visibles sont les drapeaux turcs et les statues d'Atatürk dans nombre de lieux publics tandis que les toponymes sont « turquisés ». Cette politique rencontre rapidement l'hostilité de la communauté. La tentative de remplacer les chants et danses traditionnels par des chants et danses populaires de Turquie, introduite dès la fin des années 1960, est un échec après 1974, devant la résistance de l'Association des arts populaires [Halk Sanatlari Dernegi] 5 .

En outre, la communauté chypriote turque ne partage pas le mode de vie traditionnel et parfois très religieux des colons venus d'Anatolie, ce qui engendre frictions, mépris, et incompréhensions entre les deux groupes. La communauté chypriote turque réagit à la volonté officielle d'uniformisation par le maintien de son dialecte et de ses traditions propres. L'isolement et les difficultés économiques ne favorisent pas l'épanouissement des Chypriotes turcs et certaines franges, de plus en plus larges, sont sensibles au discours de rapprochement chypriotiste. Toutefois, si le chypriotisme reflète chez les Chypriotes grecs un désir de sauver l'unité de l'île par le rapprochement entre les peuples, chez les Chypriotes turcs, il traduit plutôt une différenciation en réaction à l'assimilation turque qui leur est imposée. Il est plus souvent question pour eux de constituer une identité chypriote turque sur un territoire donné (Chypre Nord) qu'une identité chypriote globale ${ }^{6}$. Ce chypriotisme turc se traduit alors dans le discours politique par l'opposition violente entre gauche et droite nationaliste.

\section{Des rapports laborieux à l'échelle des représentations politiques}

Une première rencontre entre politiques a lieu en Libye en 1982 à laquelle participent des Chypriotes membres de l'AKEL, le premier parti de Chypre en termes électoraux, le plus favorable à un rapprochement et dont les membres

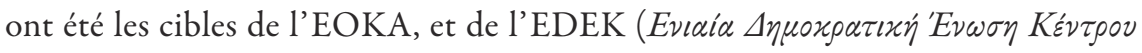

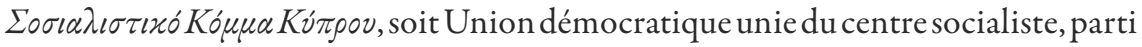
socialiste de Chypre) - un petit parti à l'origine pro-Makarios anticommuniste, mais favorable au rapprochement. Parmi les Chypriotes turcs sont présents Alpay Dürdüran, à l'époque président du TKP, parti communautaire/populaire de libération, et Özker Özgür du CTP (Parti populaire de libération). Ces deux partis sont favorables à des contacts avec les Chypriotes grecs - le CTP se dit proche de l'AKEL - mais ne sont que peu soutenus par leur opinion publique et adoptent souvent des positions pleines de réserves par rapport aux projets de rapprochement. À la fin des années 1980 et au début des années 1990, des

5. Azgin \& Papadakis, 1998, p. 703-720.

6. L'ensemble est développé dans Намiт, 2009. 
rencontres ont eu lieu au Ledra Palace entre les dirigeants des trois partis de gauche chypriotes turcs et ceux des cinq plus grands partis chypriotes grecs, mais, même si ces contacts se sont poursuivis, il est vrai qu'ils n'ont pas de résultat concret au niveau des négociations officielles.

\section{De lentes initiatives de rapprochement en parallèle des négociations au sommet}

Le déroulement des négociations officielles au début des années 1980 laisse à penser que les efforts des associations mobilisées pour le rapprochement sont vains. Elles ont néanmoins réussi à jeter des bases d'actions pour les décennies suivantes.

\section{Le rôle des syndicats}

Le dialogue entre les syndicats existait déjà avant le départ des Britanniques par le

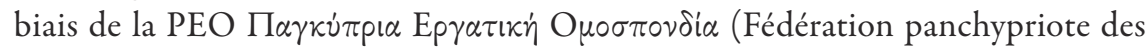
travailleurs) que les Chypriotes turcs ont dû quitter en 1958 sous la contrainte des menaces du TMT. Les syndicats chypriotes turcs sont nombreux et peu actifs hors du secteur public. Au début des années 1980 on peut dire que la Fédération des syndicats révolutionnaires (Devrimci $\dot{I}_{\xi ̧ \zeta i}$ Sendikaları Federasyonu), le syndicat des fonctionnaires de Chypre turque (Kıbris Türk amme memurlart sendikası, KTAMS), la Fédération des syndicats des travailleurs de Chypre Nord (Kıbrıs Türk iş̧i sendikaları federasyonu) ont des orientations de gauche, ce qui témoigne dans le contexte d'une volonté de jouer un rôle dans le mouvement pro-réunification. Les contacts ont repris assez rapidement après 1974, et les syndicats des deux bords suivent les négociations, allant jusqu’à faire des propositions après le set of ideas de Boutros Boutros-Ghali.

Par la suite, des réunions internationales de syndicats tenues hors de Chypre sont l'occasion de rencontres, et les contacts se renforcent grâce au All Cyprus Trade Unions Forum, qui reçoit une aide financière et politique de l'Union européenne pour ces réunions à l'étranger. La réunion de 1995 aboutit à une déclaration commune de 18 syndicats originaires des deux Chypre, celle de 1997 reprend le texte de 1995 et ajoute certains points pour préparer l'entrée de Chypre dans l'Union européenne. On y retrouve la volonté affirmée de travailler pour une Chypre fédérative et démocratique, suivant les résolutions des Nations unies, les droits de l'homme, une île qui aurait une économie unie sans aucune discrimination. Dans cet État fédéral, il y aurait un seul droit du travail, un seul système d'assurances sociales et de salaires, et l'on pourrait choisir librement son employeur n'importe où à Chypre avec liberté de mouvement. 
Les textes de 1995 et 1997 se terminent par la création d'un groupe de travail comportant des représentants des plus grands syndicats chypriotes turcs et grecs pour poursuivre le projet et une invitation pressante à tous les leaders politiques de Chypre, de l'ONU, de l'UE pour qu' ils facilitent une solution rapide et pacifique $\mathrm{du}$ «problème chypriote ». Ces réunions intersyndicales sont de loin les plus avancées, ne serait-ce que pour examiner les conséquences de l'intégration dans l'UE. Mais, bien que connues et internationales, elles sont soumises aux mêmes restrictions que les autres et, en 2002, la Fédération internationale des syndicats reçoit une plainte du syndicat SEK chypriote grec contre Rauf Denktaş qui impose des conditions totalement irréalisables aux membres chypriotes turcs du All Cyprus Trade Union Forum, afin de les empêcher de se rendre à Nicosie, précisément dans l'intention de torpiller le rapprochement.

\section{L'action des $O N G$}

Les contacts informels prennent la forme de rencontres entre universitaires, politiques, syndicalistes, associations professionnelles, le plus souvent de gauche ou du centre, ou d'associations créées par des initiatives privées hors de tout cadre officiel. Parmi les professions les plus actives, on doit indiquer les enseignants, instituteurs et professeurs d'histoire, deux des pivots de la construction identitaire d'une nation. Une partie d'entre eux sont conscients, dès les années 1970, du discours biaisé, nationaliste et porteur de haine qu'on leur fait tenir. Les syndicats d'enseignants chypriotes grecs et turcs ont des contacts très fréquents dès la fin des années 1980. Les historiens sont parvenus à créer l'Association pour le Dialogue historique et la Recherche en avril 2003, deux jours avant la première ouverture partielle de la Ligne verte; son action se déroule donc essentiellement après cette date, mais elle est multiple et trouve ses racines dans les initiatives précédentes.

Dans le même ordre d'idées, l'Institute of Historical Research for Peace (INISME), est créé en 2000. Cette ONG à but non lucratif tente de promouvoir la paix par la connaissance de l'autre, par des contacts avec celui-ci, par une meilleure connaissance de ses valeurs. Elle comprend dans ses programmes des recherches sur les relations religieuses entre musulmans et chrétiens à Chypre entre le $\mathrm{XVI}^{\mathrm{e}}$ et le $\mathrm{XXI}^{\mathrm{e}}$ siècle, et a pu programmer sur la chaîne nationale RIK 1 une émission hebdomadaire, le samedi, qui a duré sept ans : «Nos voisins et nous ». Son activité s'est encore développée au cours de la période suivante.

La plus ancienne des ONG consacrées à l'avenir de Chypre est sans doute Friends of Cyprus, créée à Londres en 1974 sous la présidence de Lord Hugh Foot Caradon, le dernier gouverneur britannique de l'île, elle comprend à l'origine des parlementaires britanniques et agit comme un lobby. Elle élargit ensuite ses contacts à d'autres groupes mais toujours des personnalités dont on suppose que 
l'action pourrait être efficace. Elle dispose à la fin des années 1990 d'un site internet complet fournissant des informations sur la politique intérieure de Chypre et de la RTCN, sur les négociations en cours entre les représentants chypriotes et l'ONU, ainsi que les textes des propositions discutées et des éléments sur les différentes étapes de l'intégration de Chypre à l'UE. Dès novembre 1974, en assemblée générale, l'organisation Friends of Cyprus se prononce pour le retrait des troupes étrangères de l'île et le retour des réfugiés. En octobre 1975, elle proteste contre la colonisation de l'île, et s'inquiète du sort des «portés disparus ». Par la suite, elle soutient toutes les résolutions de l'ONU sur Chypre et se prononce toujours pour une fédération bizonale et bicommunautaire avec retrait des troupes étrangères, ce qui, à partir de 1977, constitue un leitmotiv à Chypre.

Friends of Cyprus a été à l'origine de nombreuses rencontres intercommunautaires, mobilisant les associations de journalistes de chaque entité, des instituteurs, des ingénieurs et architectes, des médecins, ou des juristes qui étudient les systèmes des uns et des autres et leur rapport avec la législation européenne. Au cours de la dernière décennie, ces rapports se sont étoffés et constituent une revue en anglais (même si le titre est en grec et en turc) accessible en ligne. Friends of Cyprus organise, au début des années 1980, la première série de rencontres à Londres entre professionnels de la même spécialité et, très active, elle met sur pieds chaque année des manifestations suivies. Des journalistes des deux parties saisissent ainsi l'occasion de s'engager par un texte commun à bâtir la confiance entre les deux communautés, à travailler pour éliminer la suspicion envers «l'Autre » et le chauvinisme, et pour faire connaître la culture de «l'Autre »; il est même question des manuels scolaires et de leur révision nécessaire pour un regard plus pacifique. Ils demandent la création d'une carte de presse commune - qui ne sera pas acceptée - et celle, au Ledra Palace, d'un club de presse qui servirait de lieu de rencontre régulier. Tous disent être prêts à mettre en œuvre le fameux accord Denktaş-Makarios de 1977 et les 10 points de 1979, mais reconnaissent dépendre des patrons de presse et des lois de leur pays respectif.

Il faut insister sur le rôle de Londres, centre d'une importante diaspora chypriote, comme lieu de rencontre bicommunautaire. Tous les témoins précisent que dans les quartiers nord de Londres habités par les Chypriotes, Grecs et Turcs fréquentent les établissements de l'un et de l'autre dans la même rue, partagent le même CCC (Cypriot Community Center) créé au début des années 1980 avec des fonds du Harigey Center, géré par le secrétaire général de l'AKEL en Grande-Bretagne. Toutes les inscriptions sont en anglais, grec et turc, aucun symbole ni carte dans ses couloirs n'a de connotation nationaliste grecque ou turque. Des membres des deux communautés fréquentent le centre où l'on a 
résolu la question du café grec ou turc en le baptisant « café chypriote ${ }^{7}$ ». L'AKEL est très puissant parmi la diaspora londonienne chypriote, dont certains membres avaient quitté l'île bien avant 1974 en raison de poursuites politiques, et dont les récits construisent une histoire non officielle de gauche dans laquelle l'EOKA a tué plus de communistes que les Anglais; ils reconnaissent également les souffrances infligées à la communauté turque par la communauté grecque. Ce parti n'oublie pas à Londres de commémorer spécialement les assassinats de Mishaouli et de Kavazoglou un Chypriote grec et un Chypriote turc, tous deux membres de l'AKEL, tués le 11 avril 1965 par des membres du TMT.

D'autres intellectuels de gauche chypriotes grecs et turcs se sont réunis à Berlin en 1989, avec l'aide des Verts allemands, et ont créé le Mouvement pour la fédération et l'indépendance à Chypre qui ne survécut que deux ans, miné par les efforts du gouvernement de RTCN pour ne jamais permettre la sortie de son territoire à ses membres.

Un nouveau lieu est fourni par les États-Unis qui décident de reconvertir les fonds jusque-là alloués aux réfugiés à des bourses aux étudiants chypriotes des deux communautés. La Fondation Fulbright finance alors des séminaires consacrés à la résolution des conflits à partir de 1992 : elle paye des voyages, fournit des salles, règle les difficultés diplomatiques pour permettre aux chercheurs chypriotes turcs de venir. Elle invite des universitaires américains spécialisés dans les techniques de gestion et de résolution des conflits, notamment ceux de l'Institute for Multi-Track Diplomacy, du Conflict Mangement Group et du NTL Institute for Applied Behavorial Sciences ${ }^{8}$.

Les États-Unis tentent ainsi de redorer leur image et de rester présents d'une manière plus appréciée par les populations. La suite des relations montre cependant que ces initiatives nombreuses n'ont pas réussi à apporter de solution, mais leur succès a montré que la demande est forte. Les États-Unis doivent d'ailleurs demander à l'UNFICYP de céder des locaux au Ledra Palace pour ces rencontres. Il est vrai que le gouvernement américain est celui qui peut le plus influencer les autorités de la RTCN, en raison de ses liens avec la Turquie, comme bien sûr, le gouvernement turc. Les Américains tolèrent en effet la présence en RTCN de chars américains, ce que le Congrès a interdit, et en échange se voient accorder par la RTCN et la Turquie des concessions pour la circulation des militants du rapprochement ${ }^{9}$.

7. Chatzipanagiotidou, 2012, p. 86.

8. BERTRAND, 1999 , p. 31.

9. BERTRAND, 2001, p. 118-138. 
En 1998, le Département d'État américain demande à l'ONG Seeds of Peace travaillant sur le Proche-Orient de créer un programme pour Chypre. Le but est de former des «graines de paix », en réunissant des jeunes qui, dans les années 1990 et jusqu'en 2003, ne se sont jamais vus. La Commission Fulbright choisit chaque année des jeunes Grecs, Turcs et des membres des deux communautés chypriotes (80/20, le rapport en faveur des Chypriotes grecs) et organise des camps de vacances d'été dans le Maine en leur faisant découvrir l'Autre et les éléments communs de leurs héritages. Chaque jour, des instructeurs tentent de les faire sortir des stéréotypes historiques qui leur ont été inculqués et de les conduire à des visions plus nuancées. Le projet inclut également un camp dédié aux activités artistiques et une troupe de théâtre. Le site internet de Seeds leur permet ensuite de poursuivre les échanges et accueille leurs articles sur The Olive Branch, le magazine publié par Seeds à Jérusalem ${ }^{10}$.

Nous pouvons également citer parmi ces groupes actifs, le Cyprus Conflict Resolution Trainers Group (CRTG) fondé en 1994 par trente militants chypriotes pour la paix (quinze de chaque bord) qui avaient déjà largement participé à des groupes de réflexion bicommunautaires aux États-Unis et au Canada. Son travail, aidé par la Fondation Fulbright, est salué en 1997 par une résolution du Conseil de sécurité (1117, 27 juin 1997); en 1999, le programme UNOPS des Nations unies prend le groupe en charge ${ }^{11}$. L'un de ses membres fondateurs Canan Öztoprak deviendra ministre de l'Éducation dans la RTCN. Au total donc, même si les résultats ne sont pas immédiats, la plupart des activistes actuels du rapprochement ont été formés dans ces années 1980 et 1990, par ces initiatives.

\section{Les femmes en avant}

Il nous faut aussi citer des mouvements menés spécifiquement par des femmes. L'action des femmes en faveur de la paix n'est pas un phénomène chypriote, les femmes sont souvent les grandes victimes des guerres, victimes de surcroît de nombre de violences sexuelles, et souvent elles ne sont pas prises au sérieux quand elles veulent intervenir.

Les années 1990 ont vu naître une véritable action féminine à Chypre. Les femmes tentent d'imposer leur vision de la situation qu'elles appellent une culture de la solution, ou au moins d'obliger les gouvernants et négociateurs à prendre en compte leur existence dans ce qu'elles jugent, au Nord comme au Sud, être une société patriarcale, militaire et guerrière qui ne donne à la femme que le devoir

10. http://www.seedsofpeace.org/?page_id=2724\#sthash.1DehwbGX.dpuf, consulté le 23 juin 2018.

11. Anastasiou, 2008. 
de fabriquer de futurs héros et de rendre honneur aux morts selon les rites, mais aucun droit. N'oublions pas qu'aujourd'hui encore l'Assemblée chypriote compte seulement six députés femmes sur 56 et celle de la RTCN, quatre sur $50^{12}$.

Ce mouvement des femmes n'est pas uniquement chypriote, nous pouvons noter que le Conseil de sécurité par sa résolution 1325 de 2000 rappelle à ses membres les droits des femmes et leur rôle dans la paix et demande à tous ses membres d'appuyer les initiatives de paix prises par des groupes de femmes et de les faire participer aux négociations. Si peu de femmes à Chypre (et dans le monde) ont eu connaissance de cette résolution, il n'en reste pas moins que l'idée de jouer un rôle fait son chemin. Dès les années 1970, Chypre compte une Association des réfugiées femmes, plus centrée sur la douleur commune aux deux communautés et moins sur l'égoïsme de la douleur exclusive que les associations de réfugiés originaires d'un seul village.

Ce premier pas est suivi par le Women Walk Home (WWH), un mouvement apolitique de Chypriotes grecques qui demandent une île réunifiée sans Ligne verte ni armée d'occupation, une île où deux communautés coexisteraient, où elles auraient le droit de rentrer chez elles! En 1975, en juin et novembre 1987, en mars 1989, des femmes du WWH ont tenté, malgré l'UNFICYP et les troupes turques, de passer la zone de démarcation pacifiquement en tenant des banderoles en grec et en turc, sous le regard de sympathisantes étrangères venues les soutenir. Leur tactique consiste à égarer les forces réduites de l'UNFICYP en organisant de faux rassemblements très visibles en certains points ou en faisant tourner des autocars. Elles parviennent ainsi à passer là où on ne les attend pas... Elles doivent une part de leur célébrité à Titina Loïzidou, car c'est à l'occasion de la manifestation de 1989 que cette dernière, arrêtée en zone turque et remise rapidement au check-point de l'ONU à Nicosie, se décide à porter plainte auprès de la Cour européenne pour atteinte à son droit de se déplacer et de jouir de ses propriétés.

En 1989, plus de 3000 des femmes du WWH ont passé la Ligne en deux endroits (selon la même tactique), les heurts avec la police turque ont fait neuf blessés, 50 personnes ont été arrêtées puis relâchées au check-point de Nicosie. Un affrontement majeur avec la police a eu lieu dans le village de Lymbia à une vingtaine de kilomètres au sud-est de Nicosie, où les femmes étaient entrées en zone turque pour se rendre à la chapelle Saint-Georges transformée en poste de garde turc. D'un village turc voisin quelqu' un est venu avec une banderole « Plutôt séparés que morts ». À Akhna, un village voisin désert, 25 autres personnes sont arrêtées pour avoir brièvement occupé une église. Un reporter de l'Associated Press

12. Demosthenous, 2011. 
rapporte qu'une vieille femme a supplié les gendarmes turcs de la laisser voir sa maison. Rauf Denktaş se serait contenté de déclarer qu'il s'agissait d'une simple propagande nuisible aux négociations en cours.

À la même époque naît une nouvelle initiative pour favoriser les rencontres entre les femmes, grâce à l'action de Simone Susskind, présidente du Centre communautaire juif laïque de Bruxelles, qui organise en 1989 une conférence «Donner une chance à la paix, les femmes prennent la parole». Cette conférence réunit des Israéliennes et des Palestiniennes et aboutit à la création du réseau Jerusalem Link. En 1995, Simone Susskind fonde l'ONG Actions en Méditerranée; Cyprus Link est créé à Bruxelles en avril 1997, avec le soutien de l'Union européenne. Vingt-cinq Chypriotes grecques et autant de Chypriotes turques participent à la fondation de cette organisation, parmi elles, les représentantes de dix-huit ONG, sections féminines de partis politiques et de syndicats. Une deuxième conférence se tient à Londres, en mars 1998, et Cyprus Link prévoit de se transformer en ONG en 1999.

Le Bicommunal Women's Group est quant à lui, en 1995 et 1996, un mouvement plus militant. Il rassemble 22 femmes venues en nombre égal, des deux communautés, avec l'aide de la Commission Fulbright, sous la direction de Maria Hadjipavlou et de Sevgül Uludag, qui ont travaillé d'abord séparément de chaque côté de la Ligne verte, puis ensemble au Ledra Palace, après avoir obtenu des permis de passage, sur les souffrances vécues par les femmes et les réponses qui peuvent y être apportées.

Ces travaux portent notamment sur des enquêtes auprès des Chypriotes grecques et turques. L'une d'entre elles fait en particulier apparaître que les femmes chypriotes turques ont cité 82 facteurs différents de séparation, dont beaucoup autour de la notion de peur : peur d'être exclues par les Chypriotes grecques, peur de dire ce qu'il ne faut pas dire et d'être ensuite ostracisées chez elles, peur des militaires turcs et des colons turcs anatoliens, peur de l'inconnu... Elles ont même conclu qu'elles se sentaient plus « chypriotes » que les hommes, sans doute parce qu'elles reliaient le nationalisme au militarisme qu'elles rejetaient ${ }^{13}$. Certaines de ces femmes n'avaient encore jamais eu l'occasion de rencontrer des Chypriotes de l'autre partie et la plupart n'étaient pas des militantes. Ce travail d'échange est malheureusement interrompu par la suspension des permis de passage dans la zone tampon décidée par Rauf Denktaş.

L'année 1997 voit également la naissance du KAYAD Toplum Merkezi (Centre communautaire), une organisation de femmes chypriotes turques dont la présidente est Meral Akıncı, épouse de l'actuel président de la RTCN. Elle a

13. Hadjipavlou, 2006. 
pour objectif de travailler pour les droits de l'homme, et pour ceux de la femme en particulier, en renforçant la position de cette dernière dans la société, et en œuvrant pour la paix et la tolérance. Elle souhaite également promouvoir « l'unité dans la diversité $\gg$, diversité de race, de sexe, de culture, de foi, de langue. Le Centre communautaire, organise également des ateliers et des conférences, et propose des cours de grec et de turc.

Comme Meral Akıncı, Katie Cleridès, fille du président Cleridès, l'une des rares députées chypriotes grecques, œuvre au rapprochement. Elle crée en 1999 le Bureau bicommunautaire de réconciliation et de renforcement de la société civile dans le cadre du DISY. Ses membres viennent de tous les partis et des différentes communautés de l'île, Chypriotes grecs, turcs, maronites, arméniens, latins. Ils cherchent à organiser des groupes de contact entre Chypriotes et à promouvoir la réconciliation par des conférences et discussions; mais, bien que fille de Glafkos Cleridès, elle rencontre souvent des difficultés, car le DISY n'est pas particulièrement favorable au rapprochement avec les Chypriotes turcs.

Une autre initiative importante réside dans l'action de l'association de femmes Hands across the Divide (HAD), fondée en 2001 par Maria Hadjipavlou et Sevgül Uludag, avec une aide du British Council, qui a invité des Chypriotes à participer, à Londres, à une conférence rassemblant des femmes de plusieurs régions de conflit dans le monde. C'est la première association bicommunautaire chypriote reconnue au plan international. Elle multiplie les activités, interventions dans la presse, lettres aux leaders à la veille des négociations, appel au public pour déposer au cœur de Nicosie une «montagne» de messages de paix à envoyer dans le Nord. Des messages du même genre sont déposés près du domicile de Rauf Denktaş, HAD multiplie par ailleurs les interventions dans les médias, radio et télévision. Elle publie le journal HADE (Allons-y, en grec et en turc), l'un des premiers organes de presse à Chypre à avoir utilisé internet pour sa diffusion. Il a été tiré à 2000 exemplaires pour son premier numéro, avec un retirage de 1000 exemplaires supplémentaires, et à 3000 exemplaires pour le deuxième.

Compte tenu de son mode de diffusion (au porte-à-porte et de bouche à oreille en zone sud, sous le manteau en zone nord) et du fait que les fonctionnaires chypriotes turcs n'ont pas l'autorisation de participer aux activités bicommunautaires (à l'exception des représentants syndicaux), nous pouvons mesurer l'ampleur de la mobilisation en faveur de la réconciliation, ou à tout le moins, du dialogue ${ }^{14}$. C'est ce désir de paix que l'on retrouve dans cet extrait du poème de Neriman Cahit où une Chypriote turque parle à son amie Cleopatra,

14. BertRAND, 1999, p. 32. 
108 Politique et sociétés à Chypre aujourd'hui

une Chypriote grecque, tandis qu'elles joignent leurs mains pour planter des amandiers dans la zone tampon en espérant un avenir de paix pour leurs enfants.

Neriman Cahit, Nous arrivons trop, trop tard, Cleopatra (1995)

... Nous arrivons trop, trop tard, Cleopatra

regarde... regarde nos fils, regarde...

ils tiennent à nouveau des fusils,

écoute... écoute mon cri, Cleopatra, écoute... on tue nos fils

l'un, ça vient de ton côté, l'autre, de mon côté,

l'un, ça vient de ton côté, l'autre, de mon côté,

et puis des années suivent des années

des meurtres, d'autres meurtres.

Pensons pour une fois, qui est le vrai perdant,

au lieu des politiciens,

pensons-y pour une fois, nous les mères ${ }^{15}$.

Ces efforts sont-ils couronnés de succès ? Portent-ils leurs fruits alors même que l'on sait que les mentalités ne changent que lentement?

La réponse est négative, si nous considérons les résultats des négociations. Mais, en tenant compte du fait que les responsables impliqués ne changent pas, nous observons néanmoins que, malgré les difficultés, le déplacement nécessaire vers Nicosie, le contrôle des passages de la Ligne verte, le poids du qu'en-dira-t-on et la force de la propagande nationale, un groupe de 4000 à 5000 personnes au moins est impliqué. Le Ledra Palace est le lieu de réunions quasi quotidiennes à la fin des années 1990, les concerts (Burat Kut/Sakis Rouvas) et les fêtes bicommunautaires organisées par l'UNFICYP parviennent à réunir 4000 à 5000 spectateurs. Ce succès peut expliquer pourquoi Rauf Denktaş, réfractaire à l'idée d'une possible entente bicommunautaire, a supprimé les permis de passage.

\section{Les années 2000, un tournant dans les relations communautaires?}

Après trente années de négociations infructueuses, des dizaines d'initiatives de citoyens cherchant un rapprochement, deux sociétés bloquées sur leurs traumatismes, les discussions précédant l'année 2004 semblent enfin annoncer le commencement d'une issue au «problème »: référendum sur un plan de fédération proposé par le Secrétaire général des Nations unies, Kofi Annan (24 avril 2004), négociations pour l'entrée de l'île dans l'Union européenne

15. Traduction d'Alexandre Lapierre depuis le poème «Çook, çok geç kaldık, Kleopatra» de Neriman Cahit. 
(effective le $1^{\text {er }}$ mai 2004), et, liée à cette entrée, ouverture déjà entamée de la Ligne verte, un premier pas lourd de conséquences effectué en avril 2003, dans l'optique de l'acceptation du plan Annan espérée par les Chypriotes turcs.

L'ouverture du check-point central de Nicosie fermé - sauf rares exceptions depuis 1974 à tous les Chypriotes est un réel choc, - émotion lors du passage, nouvelles émotions en revoyant des paysages perdus, accueil enthousiaste des premiers Chypriotes turcs qui virent les Chypriotes grecs. Ouverture physique et symbolique, elle contribue à changer le cadre psychologique des relations entre les hommes ${ }^{16}$. Des Chypriotes patientent jusqu'à 12 heures dans leur voiture pour passer par le seul point ouvert en avril 2003 le premier jour. 350000 personnes sont passées en 3 jours et plus de 3 millions en une année. De janvier à mai 2015, 868694 traversées ont été enregistrées, 951081 dans la même période en 2016, 805269 du 24 juin 2016 à la fin du mois de novembre de la même année.

Cette conjoncture nouvelle, l'avènement au niveau dirigeant d'une génération née après 1974, et les efforts faits par les partisans du rapprochement, en particulier dans le domaine éducatif, peuvent-ils débloquer une situation gelée depuis si longtemps? Un possible accord fait intervenir de multiples acteurs qui sont loin d'être unanimes: les Chypriotes - leurs dirigeants, l'opinion publique et les activistes des associations favorables au rapprochement - et les « puissances garantes », la Turquie, la Grèce et la Grande-Bretagne. Là aussi, dans chaque pays, les politiques des différentes tendances, les religieux, les nationalistes, l'opinion publique, ont leur influence. L'ensemble de ces acteurs se trouve de surcroît inclus dans le contexte économique, politique et stratégique du Proche-Orient dont ils doivent tenir compte. Les acteurs et les éléments de contexte sont nombreux, multipliant les points d'achoppement et rendant les perspectives de rapprochement incertaines. Nous pouvons constater que si la décennie ouverte par les négociations de 2003-2004 est marquée par une certaine inertie des pourparlers au sommet, des changements concrets se produisent sur le terrain. La relative accélération des négociations voulue par les dirigeants chypriotes grec et turc depuis 2015 s' inscrit dans ce contexte interne sans doute plus favorable. Le changement de génération, à Chypre comme en Turquie et en Grèce, le contexte nouveau en Méditerranée orientale, contribuent depuis trois ans à une accélération nette du mouvement vers l'entente, mais les blocages ne disparaissent pas pour autant.

\section{Le rôle des Nations unies}

Les Nations unies sont présentes dans l'île depuis 1964 à travers la Force des Nations unies à Chypre (UNFICYP) dont le mandat est toujours régulièrement

16. Demetriou, 2007, p. 987-1006. 
reconduit. L'efficacité de cette politique de pacification reste discutable dans la mesure où elle n'est pas parvenue, faute d'un mandat suffisamment large, à contrer les événements de 1974 ni à répondre aux graves troubles qui ont opposé les deux communautés en 1996 (affaire Tassos Isaac). Depuis 1974, cinq Secrétaires généraux se sont penchés sur le sort de Chypre. Parmi eux, quatre ont proposé des plans ambitieux afin de solder la discorde intercommunautaire, mais ni Kurt Waldheim, ni Javier Pérez de Cuéllar, ni Boutros Boutros-Ghali, ni Kofi Annan ne sont parvenus à concilier des positions incompatibles, essuyant souvent des refus de dernière minute. Ban Ki-Moon, le dernier des cinq, a missionné un haut représentant sur place, l'Australien Alexander Downer puis en août 2014 un Norvégien, Espen Barth Eide, afin d'inciter les deux parties à négocier sur la base du dernier plan proposé, le plan Annan. Ban Ki-Moon multiplie en outre les contacts personnels directs avec les dirigeants chypriotes des deux bords.

La présentation des initiatives successives des Nations unies à Chypre s'apparente donc à un exercice peu efficace, car elles butent toujours sur les mêmes difficultés ${ }^{17}$. Après une reprise en juillet 1997, en effet, les négociations se sont multipliées sans succès. En 2000, au quatrième tour des discussions à New York, comme dans un cinquième tour à Genève, les propositions du Secrétaire général sont rejetées par les deux parties. Les uns refusent l'idée d'une Confédération, les autres celle de libre circulation et de libre établissement des Chypriotes sur toute l'île.

Il peut sembler paradoxal de présenter l'année 2004 comme un tournant puisque c'est celle de l'échec du nouveau plan parrainé par le Secrétaire général des Nations unies, Koffi Annan; cet échec a néanmoins contribué à faire avancer la réflexion des parties concernées et à débloquer partiellement la situation. Les négociations sous l'égide de l'ONU ont donné lieu à une forte mobilisation des opinions publiques de part et d'autre de la Ligne verte, en particulier de la part des Chypriotes turcs contre le régime de Rauf Denktaş, conduisant à des décisions politiques marquantes comme l'ouverture unilatérale par la partie turque de la Ligne en avril 2003.

\section{L'action des organisations non gouvernementales et des associations dans la révision des mythes nationaux}

Au début des années 2000, un changement de personnel politique (la mort de Turgut Özal, puis celle d'Andréas Papandréou), l'action de la société civile, les pressions des hommes d'affaires, couronnés par le choc émotionnel des deux 
séismes de 1999 à Athènes et Izmit incitent les gouvernements grec et turc à conclure une série d'accords et à prévoir de « réviser » leurs manuels scolaires, accusés de répandre la haine et la peur de l'Autre. Précisons que dans les deux pays, le manuel est unique, choisi par le ministère de l'Éducation, et que la récitation «par cœur» reste primordiale, d'où l'importance de ces textes. Le manuel d'histoire est spécifiquement concerné puisque nul n'ignore son importance pour édifier et diffuser le récit national indispensable à la cohésion d'un État.

Ce contexte n'est pas sans conséquence pour les Chypriotes dont les communautés grecque ou turque utilisent le manuel en vigueur en Grèce ou en Turquie. C'est donc entre 2002 et 2005, dans le contexte des discussions autour de l'entrée de Chypre dans l'Union européenne et du plan Annan que naît dans l'île la majorité des initiatives, après une période de maturation qui commence en 1999 chez les Chypriotes turcs. Un tournant se produit en 2003 lorsqu'en RTCN le parti nationaliste perd les élections au profit du Parti républicain turc (CTP) qui s'attaque immédiatement à la révision des manuels, pour diminuer l'insistance sur les «Turcs » au profit des «Chypriotes ». Parmi les organisations dont les travaux ont été utilisés ici, on trouve ceux de plusieurs associations très actives:

- Le CRDSEE (Center for Democracy and Reconciliation in Southeast Europe), créé en 1998 par un groupe d'hommes d'affaires et de diplomates originaires des Balkans et de Turquie. Parmi ses nombreuses actions en faveur de la paix, il se tourne vers l'enseignement de l'histoire en privilégiant les initiatives pluralistes. Ce travail donne lieu à la publication en 2002 à Thessalonique, sous la direction de Christina Koulouri, de Clio in the Balkans, un ouvrage qui regroupe des contributions sur l'enseignement de l'histoire, les manuels en usage et les stéréotypes en vigueur dans chacun des pays concernés. Christina Koulouri avait déjà, entre 1996 et 2000, été membre du projet financé par le Conseil de l'Europe «Apprendre et enseigner l'histoire de l'Europe au $\mathrm{XX}^{\mathrm{e}}$ siècle » et du groupe gréco-turc « Projet historique partagé » qui étudiait la question toujours épineuse entre les deux pays de l'incendie de Smyrne en 1922. Le CRDSEE finance également la publication d'une série d'ouvrages sur l'histoire des Balkans, toujours édités par Christina Koulouri. La collection, intitulée "Teaching Modern Southeast European History: alternatives educational materials", comprend aujourd'hui quatre titres: L'Empire ottoman, Nations et États en Europe du Sud-Est, Les Guerres balkaniques et La Seconde Guerre mondiale. Enfin, Christina Koulouri a coordonné en 2011 une équipe de cinq historiens des Balkans pour l'exposition qui 
eut lieu à l'Institut Goethe « Récit balkanique » (photos des monuments ottomans, catalogue, site internet et manuels éducatifs).

- Le mouvement Education for Peace, né en 2000, lance un programme dans six écoles de Bosnie-Herzégovine pour « créer une culture de la paix » dans cette région au sortir de la guerre. D'une phase à l'autre, il fait participer un nombre grandissant d'écoles en Bosnie et étend son activité à d'autres régions du monde. À Chypre, dans sa deuxième phase (2004-2009) il soutient et étudie les nouveaux manuels d'histoire chypriotes turcs, dans la phase suivante, il compare les manuels de 2009 et ceux de $2004^{18}$. La publication des travaux est soutenue par l'UE, l'UNDP-ACT/USAID et le POST RI.

- Le POST RI (Research Institute) est créé en 2002 par un groupe d'intellectuels chypriotes soucieux d'《éclairer» la société civile pour réduire l'incompréhension entre communautés. Ils collaborent avec Education for Peace dans le domaine scolaire, et réalisent de nombreux ateliers, voyages, visites et contacts extérieurs. Un projet actuel (2014-2017) regroupe Education for Peace, le POST RI et l'Association pour le dialogue et la recherche historique (ADRH), née en 2003, avec le soutien de la Commission européenne. Il s'agit toujours de chercher par l'éducation à changer la culture de conflit qui domine encore le discours chypriote en s'adressant aux enseignants.

- L'ADRH réunit des universitaires et enseignants chypriotes grecs et turcs et l'université de Cambridge. Depuis 2010, elle actionne un « Projet de dialogue interculturel sur l'éducation $\gg$. L'ensemble s'intègre dans un programme d'action des Nations unies pour la coopération et la confiance (UNDP-ACT) et reçoit l'aide de l'Union européenne. Son but est de promouvoir une vision pluraliste de l'histoire de Chypre et d'aider les enseignants en leur fournissant des documents sur certains points cruciaux. Elle tient aussi à Nicosie, avec le soutien du Conseil de l'Europe, plusieurs séminaires sur l'enseignement de l'histoire, les manuels et les moyens d'atteindre la «pluri-perspective » dans un milieu qui souvent ne lui est pas favorable. L'étude publiée en 2011 sur «Les enseignants d'histoire dans les communautés chypriotes grecque et turque à Chypre (perceptions, croyances, pratiques) » montre l'intérêt et le besoin ressenti par ces personnels. L'enquête a porté sur 5357 enseignants du primaire (dont 4082 Chypriotes grecs) et 1715 enseignants du secondaire (dont 1597 Chypriotes grecs), sur leur attitude face à leur enseignement, leurs

18. Beyidoglu et al., 2010. 
besoins en spécialisation et leurs convictions sur les grandes questions des rapports gréco-turcs et des relations intracommunautaires. Dans l'ensemble, la majorité d'entre eux, Chypriotes grecs comme turcs, n'ont pas fait d'études d'histoire, déclarent ne pas se sentir parfaitement à l'aise dans leur enseignement et être prêts à suivre des séminaires de complément, ce qui n'est arrivé qu'à un très faible pourcentage d'entre eux, car souvent les séminaires existants ont lieu à l'étranger.

C'est donc pour former les enseignants à des méthodes et à un contenu plus complet, tout en leur laissant une grande souplesse d'utilisation, que l'ADRH a édité cinq ouvrages trilingues (anglais, turc, grec): La période historique à Chypre, Penser historiquement la question des personnes disparues à Chypre, Apprendre à interroger l'histoire de Chypre à travers ses artefacts (sur l'emploi des illustrations et des musées), Introduction à l'histoire orale et Nos enfants - nos jeux. Certains livrets portent sur des sujets plus sensibles que d'autres, ainsi l'histoire ottomane et la question des portés disparus. Chaque livret fournit des extraits de textes et des photos puis de possibles questions analysant le document. Le guide portant sur l'enseignement des personnes disparues reflète le côté «difficile » du sujet: il comprend six fascicules (tous les autres, un seul) et trois DVD. L'ensemble est conçu pour être utilisé au besoin en dehors de la classe dans les associations ou groupes de jeunes. Il tente de dédramatiser le sujet en le replaçant dans un cadre international et en se posant la question du souvenir, de la mémoire à perpétuer et des modalités de cette perpétuation.

L'ADRH a également publié en 2011 un livret sur Les anciens villages mixtes de Chypre, représentations du passé, du présent et du futur. Il s'adresse à une génération qui, - y compris les enseignants - est née après 1974 et n'a jamais connu ces villages mixtes, leur organisation, et les rapports entre les villageois des deux communautés que par oui-dire ou par le discours officiel de chaque communauté. Le travail fourni se fonde sur des entretiens avec 1005 Chypriotes grecs et 882 Chypriotes turcs de plus de 60 ans, des hommes à $55 \%$. L'ADHR dispose depuis 2011 d'un centre, Home for Cooperation, une ancienne demeure arménienne située en face du Ledra Palace, acquise grâce à l'aide de la Norvège, de l'Islande, du Liechtenstein, de la Suisse, des Pays-Bas, de l'UNDP-ACT et d'USAID. Ce centre plurifonctionnel a pour objectif de promouvoir le dialogue et la coopération en étudiant les plans ethniques, religieux, linguistiques et culturels différents au niveau local, régional, européen et international.

- Un groupe de sept chercheuses chypriotes grecques, à la demande du ministère de l'Éducation de Chypre, avec le soutien des Nations unies, 
entreprend en 2003 une étude des manuels d'histoire et de littérature du CM2 chypriote grec $^{19}$.

- Depuis 2005, le centre chypriote PRIO Cyprus (antenne du PRIO Peace Research Institute Oslo) regroupe des chercheurs chypriotes grecs et turcs qui, eux aussi, cherchent à faciliter le rapprochement et l'unité et collaborent avec l'ADRH dans leurs travaux éducatifs ${ }^{20}$.

Deux associations d'enseignants (du primaire et du secondaire) de la RTCN (KTÖS et KTÖES) entretiennent des contacts étroits avec leurs homologues chypriotes grecs et l'United Cyprus Platform à Istanbul grâce aux associations chypriotes de l'étranger.

Les enseignants chypriotes grecs ou turcs qui ont étudié leurs manuels en vue d'une possible réconciliation y ont vu les mêmes défauts, nationalisme exacerbé, refus de l'Autre, incitation à la haine, absence d'esprit critique. Ces réflexions s'inscrivent dans le prolongement des travaux des ONG apparues dans les années 2000 à l'initiative d'universitaires ou d'enseignants, pour développer une politique de réconciliation s'appuyant en particulier sur une révision des modes d'enseignement de l'histoire. Ces organisations sont en effet conscientes que le manuel n'est pas seul à former les enfants : il faut compter avec l'enseignant, avec l'entourage familial et local et la doxa diffusée par les médias ${ }^{21}$.

Ces organisations non gouvernementales ont su acquérir le soutien, non seulement de simples particuliers et de chefs d'entreprises, mais aussi de certains gouvernements, de l'Union européenne, du Conseil de l'Europe ou d'organismes des Nations unies. Cet effort est en lien avec le contexte politique: la fin des combats des années 1990 en ex-Yougoslavie où naissent le «Centre pour la démocratie et la réconciliation en Europe du Sud-Est », en 1998, et Education for Peace en 2000.

\section{Une difficile traduction politique}

L'expérience grecque montre que la réforme des programmes d'histoire n'est pas chose facile. Il n'est pas inutile de rappeler brièvement ici l'affaire du manuel d'histoire grec. Le ministère grec de l'Éducation et des Cultes décide, au début des années 2000, après que la révision des manuels a figuré à l'agenda de la Grèce et de la Turquie, à la suite de la rencontre de Davos en janvier 1988 entre

19. Anagnostopoulou \& Michaïlidou, 2004.

20. PAPAdakis, 2008.

21. Makriyanni \& Psaltis, 2007, p. 43-70. 
Andréas Papandréou et Turgut Özal, de faire rédiger par une équipe nouvelle un manuel de CM2 qui porte sur l'histoire grecque depuis la conquête ottomane; il choisit donc une commission d'historiens, souvent formés en France, chargés de la rédaction ${ }^{22}$. En insistant sur l'action de Venizélos et d'Atatürk qui ont été capables en 1930 et 1933, malgré un coût politique élevé dans le cas de Venizélos, de construire une entente à peine dix ans après une guerre terrible qui les avait opposés, en insistant dans le cas de Chypre sur les insurrections paysannes unissant les orthodoxes et les musulmans contre le pouvoir, il y avait de quoi changer l'atmosphère régnant jusque-là.

L'ouvrage est approuvé et distribué pour l'année scolaire 2006-2007. Il soulève alors un tollé général : associations de parents, d'enseignants, de réfugiés, sociétés d'érudits locaux, associations de la diaspora, métropolites, tous se déchaînent contre ce livre «traître à la nation ». L'extrême droite organise un rassemblement le 25 mars 2007 sur la place de la Constitution à Athènes et brûle publiquement le livre. L'Académie d'Athènes propose une centaine de corrections qui sont acceptées par les auteurs, mais rien n'y fait. Le manuel est retiré par le ministère et l'on reprend le précédent. Les points litigieux concernent la vision des Turcs. Le manuel est accusé d'indulgence coupable à leur encontre dans le but de favoriser les relations gréco-turques. Il est coupable d'avoir éliminé une partie des souffrances grecques pendant l'époque ottomane (et le mythe de l'école secrète), d'avoir passé sous silence plusieurs des hauts faits des Grecs et des religieux entre 1821 et 1830 (partie qui auparavant occupait une large part du manuel), d'avoir quasiment éliminé le récit tragique de l'incendie de Smyrne en septembre 1922.

Passer d'une vision romantique, héroïque et romancée des hauts faits à une vision volontairement consensuelle. conforme aux affirmations qu'écrivent bien des historiens grecs depuis vingt ans, mais... qui restent des affirmations de spécialistes entre eux, est difficile. On peut préciser que du côté turc, les historiens réformateurs de la même période n'ont pas non plus obtenu de nouveau manuel. Une fois les mythes fondateurs devenus «vérité », il est difficile de faire marche arrière.

Les efforts des enseignants chypriotes rencontrent, eux aussi, des difficultés. Le séminaire, organisé par le Conseil de l'Europe et l'Association des enseignants chypriotes turcs, consacré le 25 novembre 2004 aux « Livres d'histoire, matériel éducatif et leur utilisation dans la salle de classe », s'adresse à ceux que l'on espère voir un jour enseigner une histoire dépassionnée, accessible aux deux communautés. Il leur est fourni des exemples européens de pays qui ont dû résoudre, eux aussi, le

22. Pour les meilleures études critiques des manuels grecs, cf. Frangoudaki \& Dragona, 1997 ; Millas, 2001. 
problème du multiculturalisme ${ }^{23}$. Trois ateliers suivent ce séminaire et montrent une demande forte des enseignants décidés face à un public disposant d'un acquis totalement différent. Il est demandé aux élèves de parler d'une «personnalité connue ». Les jeunes Chypriotes turcs ou grecs ne citent pas les mêmes personnes, les enseignants interprètent différemment les conséquences d'un même événement, par exemple le débarquement à Chypre de Richard Cœur de Lion ou l'enrôlement des Chypriotes dans les armées britanniques de la Seconde Guerre mondiale... Quel rôle attribuer aux monuments historiques? Faut-il les restaurer ou les détruire? Sont-ils « historiques » pour tout le monde? C'est aussi l'objet d'un questionnement jugé utile par tous les participants. Depuis lors, les deux Associations des enseignants chypriotes turcs poursuivent ce type d'action.

Leur bonne volonté, et le changement politique de 2003 en RTCN ont fait naître très rapidement une nouvelle histoire de Chypre. Reflétant des choix politiques opposés au parti nationaliste, ce manuel d'histoire de Chypre destiné aux enfants de la RTCN ne parle plus de la Turquie comme la Mère-Patrie, mais de Chypre, non plus de Turcs, mais de Chypriotes turcs ou grecs, et il porte sur sa couverture une carte de l'île de Chypre non divisée, il comporte également de nombreuses illustrations ${ }^{24}$, insistant sur les points communs et les ressemblances entre les deux communautés, qu'elles ne présentent pas comme deux ensembles monolithiques. L'accent est mis sur des phénomènes sociaux, sur les activités et les contacts entre communautés, et la responsabilité des affrontements est portée par les nationalistes des deux bords (TMT et EOKA), la politique britannique, la Guerre froide, la presse, les politiques... Les violences ne sont plus attribuées à un seul bord. Même si les spécialistes ont pu trouver encore quelques points « imparfaits », ce manuel traduit un net revirement et un réel pas vers l'Autre qui n'a pas été effectué dans le manuel grec équivalent. L'objectif politique émergent d'une réunification, a poussé à inclure les Chypriotes grecs dans le schéma historique, l'identité n'étant plus présentée comme un fait génétique immuable, mais comme le fruit construit d'une évolution historique.

La campagne électorale de 2009 en RTCN montre toutefois la fragilité de ce changement et son lien avec la politique. Le manuel révisé de 2004 est au cœur de cette dernière. Le parti de droite UBP (Parti Unité nationale) annonce que s'il est élu, il supprimera ce manuel déclaré «loin de notre identité nationale ${ }^{25} \gg$;

\section{Conseil de l'Europe, 2004.}

24. PAPADAKis et. al. 2010, p. 1-96.

25. Beyidoglu et. al., "Textual and visual analysis of the Upper secondary school Cyprus History Textbooks, Part II, between 2004 and 2009", in BEYIDoglu et. al., 2010, p. $96-165$. 
le parti démocratique fait une annonce analogue, car c'est, dit-il, un livre de «culture » et non un livre d'histoire des Chypriotes turcs. Les partis de centre gauche CTP et DTP soutiennent le manuel de 2004. Un groupe dit «Comité technique historique du Conseil de lutte nationale » organise des rencontres avec tous les hommes politiques pour les persuader qu'il s'agit d'un livre qui veut faire oublier aux enfants leur turcité, en les mettant sur le même pied que les Chypriotes grecs. L'ancien ministre de l'Éducation de la RTCN riposte que c'est un livre d'historiens, et non de politiciens. Le secrétaire de l'Association des enseignants chypriotes turcs (KTÖS) estime pour sa part qu'il est inadmissible d'envisager «d'aller en arrière et d'enseigner une histoire de sang, de larmes, d'ennemis, de chauvinisme et de préjugés. » Même Rauf Denktaş se met de la partie pour déclarer que les manuels doivent être revus par un historien «nationaliste et patriote » et qu'il ne faut pas tenir compte des standards européens en ce domaine puisque l'UE cherche à couper Chypre de sa turcité. Le succès électoral de l'UBP est suivi du retrait immédiat du manuel de 2004 vite remplacé par un nouveau manuel.

Comparé au manuel de 2004, celui de 2009 tient à montrer que Chypre est une île turque (sur la couverture figurent Atatürk et trois images qui se réfèrent à l'Empire ottoman) et il est beaucoup plus turco-centré, beaucoup plus court également (109 pages au lieu de 194) que le précédent. « Chypre de la Préhistoire à la fin du Moyen Âge » y prend autant de place que «la vie du Docteur Fazil Küçük à notre Lutte nationale ». Le livre de 2004 s'efforçait de situer chaque question dans le contexte d'une histoire générale avant de passer à Chypre, celui de 2009 ne traite que de Chypre et de son caractère turc. Il innove en couvrant la période qui va de la création de la RTCN aux négociations du plan Annan et aux raisons de son rejet, en introduisant des graphiques, un peu d'histoire économique et un glossaire. Les chercheurs ont constaté que les livres de 2009 sont revenus à une vision ethnocentrée et plus militariste, y compris dans le choix des illustrations. Il revient aux notions du livre de Serter, mais avec quelques nuances, sans doute pour tenir compte du climat politique changé en RTCN : il admet une «culture chypriote turque ».

Les bonnes volontés ont du mal à s'imposer. Les aventures et conflits autour des manuels scolaires d'histoire montrent à quel point le sujet reste « un point chaud». Malgré ce relatif échec, l'intérêt des enseignants, l'activité soutenue des Associations d'enseignants chypriotes turcs, le travail des universitaires des communautés - aidés davantage par organisations internationales que par leurs gouvernements - est un exemple de l'action de la société civile en faveur d'une réconciliation. La création ou recréation d'un récit historique national reste toutefois un travail de longue durée et il n'entre pas seul en ligne de compte dans la formation des mentalités. 
Le livret de l'ADHR (2011) sur Les anciens villages mixtes de Chypre, représentations du passé, du présent et du futur, montre qu'en dehors de l'école, l'enfant rencontre d'autres formes de mémoires tout aussicontradictoires. L'enquête s'est fondée sur des entretiens avec 1005 Chypriotes grecs et 882 Chypriotes turcs de plus de 60 ans, des hommes à $55 \%$. Les réponses portant sur le passé - tel que reconstruit par les processus de la mémoire - montrent des groupes humains avec peu de relations entre eux : $31 \%$ des Chypriotes grecs et $60 \%$ des turcs disent n'avoir eu aucun ami en dehors de leur groupe, $83 \%$ des Chypriotes grecs et $63 \%$ des turcs, affirment qu' il n'y avait aucun mariage mixte (mais $12 \%$ et $31 \%$ disent le contraire!), qu'ils ne fréquentaient jamais les commerces de l'autre communauté ( $52 \%$ des Chypriotes grecs, $39 \%$ des turcs), n'allaient jamais dans un café tenu par un membre de l'autre communauté (54\% des Chypriotes grecs, $61 \%$ des turcs). On constate déjà de fortes différences entre la vision des uns et des autres. Mais certaines questions rencontrent des réponses encore plus éloignées, ainsi qui avait la plus grande force économique ou combien de membres de l'EOKA ou du TMT y avait-il au village. Tout ceci promet des difficultés aux enseignants s'ils vont contre la doxa dominante. De surcroît, entre 37 et $40 \%$ des personnes interrogées disent compter un membre de leur famille tué ou disparu en 1974. Et les questions sur la solution possible au problème des propriétés perdues en 1974 et sur l'avenir de l'île montrent le même abîme, dans certains cas, pratiquement impossible à franchir: un État unitaire est «absolument inacceptable p pour $10 \%$ des Chypriotes grecs et $63 \%$ des Chypriotes turcs, la reconnaissance de deux États également «absolument inacceptable » pour $84,7 \%$ des Chypriotes grecs et $13 \%$ des Chypriotes turcs. L'effort pédagogique à produire est donc important, et ce que cherchent les réformateurs, c'est simplement à enseigner aux enfants qu'il existe au moins deux visions, deux interprétations à prendre en compte.

En 2004, le Comité de réforme éducative de la République de Chypre, à la demande du gouvernement rédige un manifeste dans lequel il insiste sur la réorientation nécessaire de l'ensemble du système éducatif fondé sur les valeurs et le «savoir » grecs. Nous trouvons dans ce rapport pour la première fois dans le vocabulaire du ministère de l'Éducation de la République de Chypre, les termes «chypriote grec ». Il insiste en ce qui concerne l'histoire sur l'accent à mettre sur des modèles européens de coexistence pacifique, de multiculturalisme, de respect de la différence en éliminant le nationalisme. Il conseille des séminaires communs aux enseignants chypriotes grecs et turcs, demande l'établissement d'un comité mixte chypriote turc et grec pour élaborer un manuel d'histoire chypriote commun et critique l'emploi de manuels importés de Grèce ${ }^{26}$. Au total, tout ce

26. Makriyanni \& Psaltis, 2007, p. 43-70. 
que demandaient depuis plusieurs années les associations d'enseignants militant pour la réconciliation, une optique en accord complet avec les recherches de l'ADHR, est donc accepté.

Il est cependant difficile de passer à la pratique. En mars 2009, Andreas Dimitriou, ministre de l'Éducation de la République de Chypre (de 2008 à 2011), un psychologue reconnu, conduit une grande réforme de l'éducation chypriote à tous niveaux. Cette dernière porte également sur l'administration culturelle, mais elle soulève la fureur des nationalistes et de l'Église orthodoxe, car elle proclame que les extrémistes greco-chypriotes portent une responsabilité dans la division de l'île. Andreas Dimitriou appelle en outre à une réécriture des manuels décrivant à l'époque les Chypriotes turcs comme des barbares responsables de tous les maux. Les réformes provoquent dans le public parfois un plein accord. D'autres observateurs émettent des critiques sur certains points, mais modérées pour ne pas faire échouer le projet en entier. Les plus radicaux rejettent formellement une tentative pour «déshelléniser» Chypre au nom d'un pseudo-multiculturalisme, invoquant une histoire riche de trois millénaires d'hellénisme. Quant au Saint Synode de l'Église orthodoxe de Chypre, il est bien sûr fort mécontent de ne pas avoir été consulté préalablement.

\section{Conclusion}

Les mouvements associatifs et initiatives non gouvernementales en faveur du rapprochement communautaire à Chypre se sont construits progressivement, d'abord en opposition aux politiques, dont les choix et la symbolique au cours des années 1970 et 1980 encourageaient la partition, pour défendre une identité chypriote. Ces initiatives se sont élargies au fil des années touchant les domaines de la mémoire collective et de la préservation du patrimoine, constituant un processus de rapprochement de facto, meilleur moyen de lutter contre les stéréotypes. La multiplication des mouvements bicommunautaires est également un fait notable. Ces groupes ne représentent pas la majorité de la population, mais constituent des «minorités agissantes » qui ont pu, depuis 30 ans, faire naître des mouvements actifs, souvent aidés par les associations chypriotes turques ou grecques de la diaspora, qui militent pour toutes les formes de rapprochement.

Les initiatives se posent désormais en aiguillon des politiques et, même si certains de ces efforts n'ont des résultats que partiels, incomplets ou discutés, ils contribuent néanmoins au rapprochement, alors même que les négociations au sommet sont plutôt inefficaces. Nous notons également que le «chypriotisme » est redéfini depuis quelques années, y compris par les partisans du rapprochement. Contestataires et partisans s'accordent toutefois sur l'idée que cette conception, en 
tant que discours promouvant l'unité et les points communs constitue la meilleure opposition aux nationalismes. En revanche, «le désir de vivre ensemble », s'il est net chez certains militants associatifs qui ont souvent payé cher leurs convictions, ne semble pas encore l'objectif de tous. Il apparaît que bien des protagonistes se contenteraient du statu quo.

Le fait même de continuer ou de prévoir d'accorder aux «minorités » des représentations institutionnelles suppose que la « nation chypriote » sera grecque ou turque selon l'entité avant même que d'être chypriote. C'est sans doute là que se situent les limites ou l'échec relatif du chypriotisme tel que le rêvaient ses partisans chypriotes turcs et grecs. Toutefois, le tournant récent pris par la République de Chypre vers une politique multiculturelle peut permettre de sortir de l'impossible dialogue bicommunautaire pour créer une identité liée au territoire commun et non plus à la seule référence à la religion et/ou à la langue.

\section{Bibliographie}

\section{Monographies et thèses}

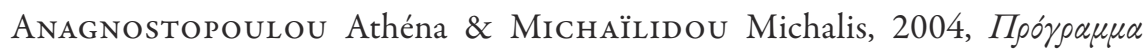

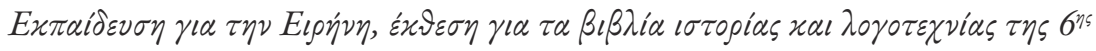

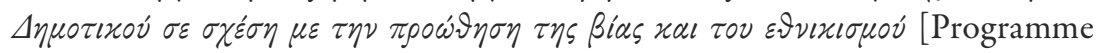
Éducation pour la Paix, exposé sur les livres d'histoire et de littérature de la $6^{\mathrm{e}}$ année du primaire, en lien avec le refus de la violence et du nationalisme], Nicosie.

Anastasiou Harry, 2008, The Broken Olive Branch: Nationalism, Ethnic Conflict and the Quest for Peace in Cyprus, Volume I: The Impasse of Ethnonationalism, Syracuse University Press, New York, 264 p.

Beyidoglu Önen, Jetha-Dagseven Shirin, Karahasan Hakan \& LATIF Dilek, 2010, Re-writing History Textbooks, History Education as a Tool for Polarisation or Reconciliation?, Tipograf Arts, Nicosia, 166 p.

Blanc Pierre, 2000, La Déchirure chypriote, géopolitique d'une île divisée, L'Harmattan, Paris, 288 p.

Chatzipanagiotidou Evropi, 2012, The Conflicts of a "Peaceful" Diaspora: Identity, Power and Peace Politics among Cypriots in the UK and Cyprus, DPhil Thesis, University of Sussex. 
Hadjipavlou Maria, 2010, Women and Change in Cyprus: Feminisms and Gender in Conflict, I B Tauris \& Co Ltd, London.

Hamit Mertkan, 2009, Cypriotism as an ideology in between Turkish and Greek nationalisms, Master, Capodistria University, Athens.

Koulouri Christina (ed.), 2002, Clio in the Balkans: The politics of History Education, CDRSS, Thessaloniki,550p,URL:http://cdrsee.org/publications/ education/clio-balkans

LAPIERRE Alexandre, 2018, Chypre: les espoirs du rapprochement communautaire, Presses de l'Inalco, Paris, $322 \mathrm{p}$.

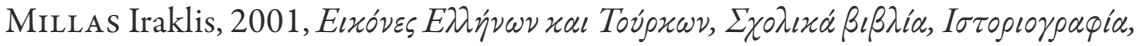

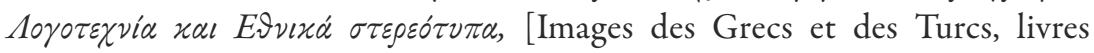
scolaires, historiographie, littérature et stéréotypes nationaux], $A \lambda \varepsilon \xi \dot{\alpha} \nu \delta \rho i \alpha$ [Alexandria] A $\theta \dot{\eta} \nu \alpha$, [Athènes].

Papadakis Yiannis, 2008, History education in divided Cyprus: a comparison of Greek Cypriot and Turkish Cypriot Schoolbooks on the "History of Cyprus", PRIO Report, Oslo.

\section{Articles et participations à des ouvrages}

Azgin Bekir \& Papadakis Yiannis, 1998, «Folklore», in Grothusen Klaus Detlev, Steffani Wienfried \& Zervakis Peter (dir.), Zeypern, Vondenhoeck \& Ruprecht, Gottingen, p. 703-720.

Bertrand Gilles, 2001, «L'adhésion de Chypre à l'Union européenne: un déblocage du conflit par le bas ? », Politique européenne, nº 3, p. 118-138.

Bertrand Gilles, 1999, «Vingt-cinq ans après, où en est la partition de Chypre? », Les Études du CERI, n 59.

Cahit Neriman, 1995, Speak now Cleopatra, URL: http://www.havenscenter. org/files/mariahadjipavlou3.pdf (consulté le 20 juin 2018).

Calotychos Vangelis, 1998, "Interdisciplinary Perspectives", in Calotychos Vangelis (ed.), Cyprus and its People, Nation, Identity and 
122 Politique et sociétés à Chypre aujourd'hui

Experience in an Unimaginable Community, 1955-1997, Westview Press, Boulder, $344 \mathrm{p}$.

Conseil De L'Europe/Association des enseignants CHypriotes TURCS, 2004, séminaire sur "History textbooks and teaching materials and their use in the classroom", 25 novembre, p. 30-35; Workshop "New approaches to teaching history: multiperspectivity”, 26-27 novembre, p. 36-46.

Demetriou Olga, 2007, "To cross or not to cross? Subjectivization and the absent state in Cyprus", Journal of the Royal Anthropological Institute, vol. 13, p. 987-1006.

Demosthenous Areti, 2011, Women's Initiatives for Peace and Reconciliation in Cyprus, URL: http://www.upf.org/resources/speeches-and-articles/4097a-demosthenous-womens-initiatives-for-peace-and-reconciliation-in-cyprus (consulté le 20 juin 2018).

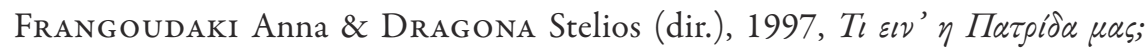

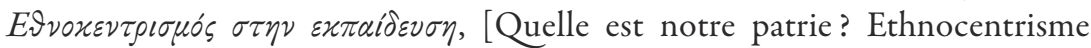
dans l'éducation], $A \lambda \varepsilon \xi \dot{\alpha} \nu \delta \rho\llcorner\alpha$ [Alexandria], $A \theta \dot{\eta} \nu \alpha$ [Athènes].

Hadjipavlou Maria, 2006, “No permission to cross: Cyprus women’s dialogue across the divide", Gender, Place and Culture. A Journal of Feminist Geography, vol. $13, \mathrm{n}^{\circ} 4$, p. 329-351.

Makriyanni Chara \& Psaltis Charis, 2007, “The teaching of History and Reconciliation”, The Cyprus Review, vol. 19, n 1, p. 43-70.

Mavratsas Caesar, 2007, "Politics, Social Memory, and Identity in Greek Cyprus since 1974", Ethnic and Racial Studies, vol. 20, n 4, p. 717-737.

Papadakis Yiannis, Karahasan Hassan \& Latif Dilek, 2010, “Textual and visual analysis of the Upper secondary school Cyprus History Textbooks", Part I, Comparative analysis of the Old and New Cyprus History Textbooks, in Re-writing History Textbooks, History Education as a Tool for Polarisation or Reconciliation?, Nicosie, POST-RI.

Résumé : Chypre vit divisée depuis 1974 sans que la succession de négociations au sommet ne soit parvenue à apporter une solution. Cet article montre que, 
néanmoins, se multiplient dans la société civile les initiatives, tant chypriotes grecques que turques, en faveur d'un rapprochement entre les communautés. Ce sont des efforts déjà anciens et qui s'accentuent depuis le début des années 2000. Les obstacles sont nombreux, dressés par les nationalistes de tout bord, un enseignement de l'histoire partisan et sans nuances, l'influence des gouvernements grec et turc, les souvenirs familiaux, etc. Pourtant, même si leur effet sur la politique est mince, chacune de ces initiatives, soutenue souvent par les institutions internationales ou européennes, des ONG, les universités étrangères, des syndicalistes, journalistes, féministes et historiens chypriotes, laisse quelques graines qui contribuent à faire évoluer lentement les mentalités.

Mots-clefs: Chypriotes, rapprochement, relations intercommunautaires, Chypriotes grecs, Chypriotes turcs, associations, ONG, histoire sociale et politique

Abstract: Cyprus has been divided since 1974 and the many summit negotiations have not brought any success. This paper shows that in the civil society, are growing initiatives, both Greek Cypriot and Turkish, in favour of a rapprochement between the communities. These are already ancient efforts that have been increasing since the beginning of the 2000's. The obstacles are numerous, drawn up by both sides nationalists, a one-sided and prejudiced history teaching, the influence of the Greek and Turkish governments, family memories, etc. Yet, even if their effect on politics is thin, each of these initiatives, often supported by international or European institutions, NGOs, foreign universities, trade unionists, journalists, feminists and Cypriot historians, leaves some seeds that help to make slowly evolve mentalities.

Keywords: Cypriots, reconciliation, intercommunal relations, Greek Cypriots, Turkish Cypriots, associations, $N G O$, social and political history

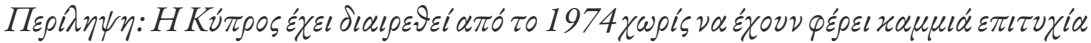

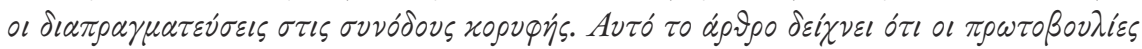

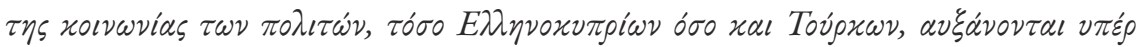

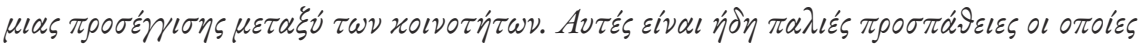

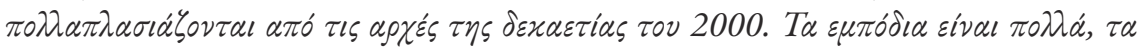

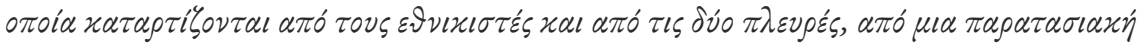

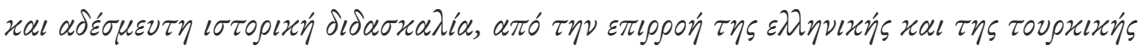

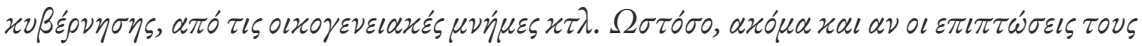

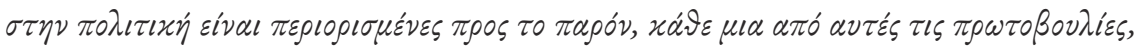

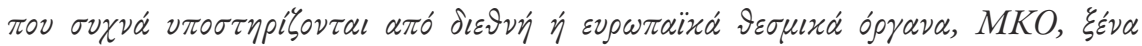

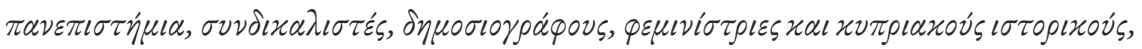

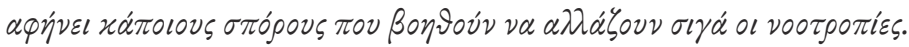




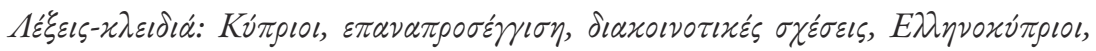

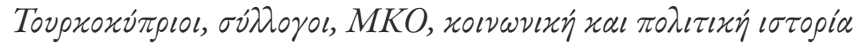

Anahtar kelimeler: Kıbrıllılar, yakınlaşma, topluluklar arası İliskiler, Kıbrıslı Rumlar, Kıbrıslı Türkler, dernekler, STK'lar, sosyal ve siyasi tarib

Клучни зборови: кипарските Грии, зближувате, односи меѓу заеднищите, кипарски Грии, кипарски Турии, здруженија, невладини организации, социјална и политичка историја 